\title{
Microanalysis of single-layer hexagonal boron nitride islands on $\operatorname{Ir}(111)$
}

\author{
Marin Petrović, ${ }^{1, \text { f }}$ Ulrich Hagemann ${ }^{2}$ Michael Horn-von Hoegen,${ }^{1}$ and Frank-J. Meyer zu Heringdorf ${ }^{1}$ \\ ${ }^{1}$ Faculty of Physics and CENIDE, University of Duisburg-Essen, Lotharstr. 1, D-47057 Duisburg, Germany \\ ${ }^{2}$ Interdisciplinary Center for Analytics on the Nanoscale (ICAN), \\ Nano Energie Technik Zentrum (NETZ), Carl-Benz-Str. 199, D-47047 Duisburg, Germany
}

\begin{abstract}
Large hexagonal boron nitride $(\mathrm{hBN})$ single-layer islands of high crystalline quality were grown on $\operatorname{Ir}(111)$ via chemical vapor deposition (CVD) and have been studied with low-energy electron microscopy (LEEM). Two types of hBN islands have been observed that structurally differ in their shape and orientation with respect to iridium, where the former greatly depends on the iridium step morphology. Photoemission electron microscopy (PEEM) and IV-LEEM spectroscopy revealed that the two island types also exhibit different work functions and bindings to iridium, which provides an explanation for differences in their shape and growth modes. In addition, various temperatures were used for the CVD synthesis of $\mathrm{hBN}$, and it was found that at temperatures higher than $\approx 950{ }^{\circ} \mathrm{C}$ boron atoms, originating either from decomposed borazine molecules or disintegrated $\mathrm{hBN}$ islands, can form additional compact reconstructed regions. The presented results are important for advancement in synthesis of high-quality hBN and other boron-based layered materials, and could therefore expedite their technological implementation.
\end{abstract}

\section{INTRODUCTION}

Alongside pioneering graphene $(\mathrm{G})$, researchers have characterized a large number of other two-dimensional (2D) materials [1] that may potentially be used in novel applications. Among them, single-layer hexagonal boron nitride $(\mathrm{hBN})$ is particularly interesting due to the isostructurality to $\mathrm{G}[2]$ and its insulating nature, i.e., a large bandgap of $\sim 6 \mathrm{eV} 3$. These properties, alongside with atomic flatness, chemical inertness and the absence of charge traps, designate $\mathrm{hBN}$ as a very important 2D material, ideal for the formation of heterostructures with $\mathrm{G}$ that promise various technological advancements, for example, in transistor [4, 5] or integrated circuits 6] fabrication. Besides in combination with graphene, hBN may be used for encapsulation of other 2D materials which enhances their performance in various devices [7]. Furthermore, other applications of $\mathrm{hBN}$ include use as an active laser medium [8] and an ultra-thin insulating support for molecular self-assembly 9, 10.

Single layers of $\mathrm{hBN}$ can be produced via chemical vapor deposition (CVD) on various single-crystal metal substrates [11 18]. Similar to the case of epitaxial G [19, 20], some metals are characterized by strong interaction with $\mathrm{hBN}$, such as $\mathrm{Ru}(0001)$ or $\mathrm{Rh}(111)$, while on other substrates hBN is more weakly bound, e.g., on $\mathrm{Pt}(111)$ or $\mathrm{Cu}(111)$ 21 23. Optimal hBN production requires a substrate to which, on the one hand, hBN binds sufficiently strongly to enable the growth of uniformly oriented domains and on the other hand, does not bind too strongly in order to preserve its intrinsic properties. These requirements are fulfilled for $\mathrm{hBN}$ on $\operatorname{Ir}(111)$ $[\mathrm{hBN} / \operatorname{Ir}(111)]$ because there the interaction is characterized as intermediate [24, 25]. Moreover, high-quality G samples with preserved intrinsic electronic structure are easily synthesized on $\operatorname{Ir}(111)$ [26] and are a very good starting point for the fabrication of G-based heterostructures. Therefore, $\operatorname{Ir}(111)$ is a suitable substrate for studying both fundamental properties of $\mathrm{hBN}$ as well as more complex systems comprised of hBN and other 2D materials.

A number of studies of $\mathrm{hBN} / \mathrm{Ir}(111)$ have been conducted so far, but there are still aspects of the system that require further investigation. For example, $\mathrm{hBN}$ exhibits two preferential orientations with respect to $\operatorname{Ir}(111)$ which were identified via X-ray photoelectron diffraction (XPD) [27] and scanning tunneling microscopy (STM) [28, but a more detailed morphological characterization of the respective hBN domains is lacking, especially on the micro-scale. Also, differences in the electronic structure of the two hBN orientations are not known, since only area-averaging ARPES has been performed for valence band mapping [29. In addition, the above mentioned studies report on the decomposition of hBN at elevated temperatures and emergence of other boron species on the iridium surface, as evidenced by STM imaging [28] as well as disappearance and appearance of different B 1s peaks in XPS [29]. Details of these processes, which are vital for establishment of highquality hBN synthesis, are not known. Moreover, insight into formation of various boron structures could initiate the production of borophene-an exciting $2 \mathrm{D}$ boron analogue of G 30]-on iridium. To investigate all these issues, the use of a complementary microscopic technique is necessary. Low-energy electron microscopy (LEEM) is an ideal tool for this task because it enables in situ, real-time observation of growth-related phenomena with the spatial resolution in the nanometer range. With this method, hBN has been characterized on e.g., $\mathrm{Pt}(111)$ [14, 31, $\mathrm{Ru}(0001)$ 32, 33] and $\mathrm{Co}(0001)$ [34, but similar studies addressing $\mathrm{hBN} / \mathrm{Ir}(111)$ are still lacking. The aim of this work is to provide first microscopic insights into this epitaxial system primarily via LEEM and in 
conjunction with several other methods, which will supplement our findings. In this way we tackle challenges of CVD synthesis of $\mathrm{hBN}$, which is the most promising technique for the large-scale production of hBN.

\section{EXPERIMENTAL}

Growth of hBN was conducted in an ultra-high vacuum (UHV) setup with a base pressure better than $3 \times 10^{-10}$ mbar. CVD was used for the synthesis of hBN during which a hot (111) surface of Ir single-crystal (Mateck) was exposed to borazine $\left(\mathrm{B}_{3} \mathrm{H}_{6} \mathrm{~N}_{3}\right.$, Katchem). The crystal was cleaned by $\mathrm{Ar}^{+}$sputtering at $2 \mathrm{keV}$ followed by several cycles of heating in oxygen at $900{ }^{\circ} \mathrm{C}$ and annealing at $1200{ }^{\circ} \mathrm{C}$. In the case when only $\mathrm{hBN}$ had to be removed from the surface, heating in oxygen and annealing were sufficient. The sample temperature was measured with an infrared pyrometer. Borazine was kept in a home-built Peltier cooler, which enabled realization of the sub-zero temperatures needed to prevent borazine decomposition. Thus, borazine was available at all times at the UHV setup, and prior to each hBN synthesis the borazine inlet line was pumped down to remove any decomposition residues. Unless otherwise noted, borazine pressure during CVD was $10^{-8}$ mbar.

LEEM and PEEM measurements were carried out in situ with an Elmitec SPE-LEEM III microscope at the University of Duisburg-Essen. PEEM measurements were performed with the aid of an Hg discharge lamp providing $\sim 4.9 \mathrm{eV}$ photons that were relevant for our samples. The spatial resolution of the microscope is $\sim 10 \mathrm{~nm}$. Atomic force microscope (AFM) measurements were performed ex situ in air with a Veeco Dimension 3100 microscope operated in tapping mode. An Auger Nanoprobe 710 from Ulvac-Phi located at the ICAN center in Duisburg was used for ex situ Auger electron spectroscopy (AES) and scanning electron microscopy (SEM) with a base pressure of $6 \times 10^{-10}$ mbar. Primary electron energy for AES was $10 \mathrm{keV}$ and $5 \mathrm{keV}$ for SEM.

\section{RESULTS AND DISCUSSION}

\section{A. Temperature-dependent growth}

In order to investigate different growth conditions, $\mathrm{hBN}$ islands were synthesized at various temperatures in LEEM. Fig. 1(a)-(c) show characteristic island morphologies for the growth temperatures of 800, 900 and $1100{ }^{\circ} \mathrm{C}$ (note that fields of view are not the same in all panels). In all cases, island nucleation was initiated at Ir step edges. At low synthesis temperatures, this resulted in the formation of strings of hBN islands that decorate the same Ir step edge, as marked in Fig. 1(a) with a yellow rectangle. The corresponding island densities in
Fig. 1(a)-(c) are 8.09, 0.58 and $0.02 \mu \mathrm{m}^{-2}$, thus spanning several orders of magnitude. At the same time, the average island size increases with synthesis temperature. These findings are in line with published STM data of hBN synthesized at different temperatures [28] and the temperature- and dose-dependence of the classical nucleation theory in general. With the growth conditions we used, hBN islands with sizes up to $\sim 10 \mu \mathrm{m}$ (for $1100{ }^{\circ} \mathrm{C}$ synthesis) were readily obtained. During prolonged exposure to borazine, individual islands coalesce, resulting in a full coverage of $\mathrm{hBN}$ and suppression of multilayer growth, similar to hBN growth on $\mathrm{Ru}(0001)$ [33].

Upon cooldown to room temperature, the formation of wrinkles was not observed on either islands or a full hBN monolayer [see Fig. 44(a) or (d) as an example]. However, wrinkling was found before for hBN on weakly interacting substrates, such as $\mathrm{Pt}(111)$ 31 or $\mathrm{Cu}$ foils 35. In contrast to hBN, G wrinkles on $\operatorname{Ir}(111)$ are routinely observed via LEEM and have been characterized in detail [36, 37]. Therefore, our data indicates that wrinkling does not occur on $\mathrm{hBN} / \operatorname{Ir}(111)$. This suggests that (i) the binding of $\mathrm{hBN}$ and $\mathrm{G}$ to Ir is different and/or that (ii) the elastic stiffness of $\mathrm{hBN}$ is lower than that of G. In comparison, theoretical calculations give binding strength to Ir of $138 \mathrm{meV}$ per $\mathrm{G}$ and $170.8 \mathrm{meV}$ per hBN unit cell [28. Also, AFM indentation experiments of suspended layers provided an elastic stiffness value of $340 \mathrm{Nm}^{-1}$ for $\mathrm{G}$ [38] and, in combination with theoretical modeling, $292 \mathrm{Nm}^{-1}$ for $\mathrm{hBN}$ [39]. Because binding and stiffness values are comparably close, it seems that a combination of both contributes to the absence of wrinkle formation in hBN.

A characteristic low-energy electron diffraction (LEED) pattern of several coalescing $\mathrm{hBN}$ islands synthesized at $900{ }^{\circ} \mathrm{C}$ is shown in Fig. 11(d). The most prominent features of the pattern are diffraction spots that reflect the presence of the moiré structure of $\mathrm{hBN} / \operatorname{Ir}(111)$ arising from the mismatch of the two surface lattices. The high quality of our hBN islands is confirmed by a large number of sharp moiré spots, which range up to the sixth order and thus span almost the entire first Brillouin zone. A closer look at the diffraction spots in Fig. 11(d) reveals that the hBN area under examination consists of several slightly rotated domains (see Subsection 3.2. for details on rotational registry of hBN and Ir). From a more detailed analysis of similar LEED patterns, we calculate the ratio of hBN and $\mathrm{Ir}$ reciprocal lattice vector modules, $k_{\mathrm{hBN}} / k_{\mathrm{Ir}}$, to be $(1.09 \pm 0.01)$. Hence, the structure of $\mathrm{hBN}$ on $\mathrm{Ir}$ is such that $\sim 10.9$ unit cells of hBN are superposed on top of 10 unit cells of Ir. The calculated ratio of 1.09 matches the ratio of $11.7 / 10.7$ (and also $12 / 11$ as the best commensurate approximation), which was recently reported as an exact description of the $\mathrm{hBN} / \operatorname{Ir}(111)$ moiré structure [28]. Knowing that the Ir real space lattice constant is $a_{\text {Ir }}=2.715 \AA$ [40], we calculate the 

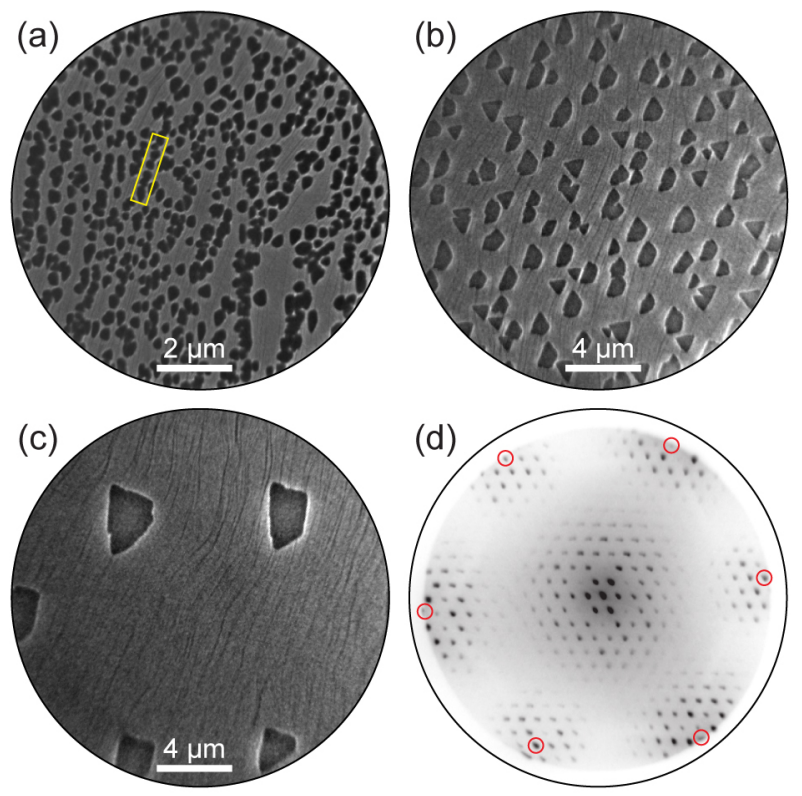

Figure 1. (a)-(c) LEEM images of hBN islands synthesized at 800,900 and $1100^{\circ} \mathrm{C}$ after $100 \mathrm{~s}$ of borazine dosing at $5 \times 10^{-8}$, $5 \times 10^{-8}$ and $3 \times 10^{-8} \mathrm{mbar}$, respectively. Images are recorded at the given synthesis temperature. $\mathrm{E}=17 \mathrm{eV}$. (d) Characteristic LEED pattern originating from several coalescing hBN islands synthesized at $900{ }^{\circ} \mathrm{C}$. Red circles indicate positions of first-order hBN spots. The image was recorded at room temperature. $\mathrm{E}=31 \mathrm{eV}$.

lattice constant of $\mathrm{hBN}$ to be $a_{\mathrm{hBN}}=(2.50 \pm 0.02) \AA$, which is in good agreement with the published values for $\mathrm{hBN} / \operatorname{Ir}(111)$ of $2.483 \AA$ [28] as well as that for bulk $\mathrm{hBN}$ of $2.505 \AA$ [41]. Also, the $k_{\mathrm{hBN}} / k_{\mathrm{Ir}}$ ratio enables calculation of the corresponding moiré periodicity, which turns out to be $a_{\text {moiré }}=(30 \pm 3) \AA$ (for the $\mathrm{hBN}$ aligned to Ir), being consistent with the reported STM measurements 28, 42.

The stability of hBN islands was examined by keeping the sample at high temperatures after stopping the borazine dosage. It was found that in the case of sufficiently high temperatures, synthesized hBN islands disintegrate from the perimeter inward and eventually vanish from the field of view. An example of disintegration is shown in a sequence of LEEM images in Fig. 22(a) for the sample temperature of $1100{ }^{\circ} \mathrm{C}$. There are two possible routes for the $\mathrm{B}$ and $\mathrm{N}$ atoms after an $\mathrm{hBN}$ island decomposes: the atoms can desorb from the sample or they can remain on the Ir surface, either in the adsorbed or dissolved form. $\mathrm{N}$ desorption and $\mathrm{B}$ dissolution into the bulk seem to be the most probable scenario because $\mathrm{N}_{2}$ molecules desorb from the (111) surface of Ir even below room temperature [43, while iridium borides of various stoichiometric ratios can be formed at high temperatures 44. Further experiments corroborating this assumption are described in the following.

Fig. 2(b) shows the surface of the sample after hBN
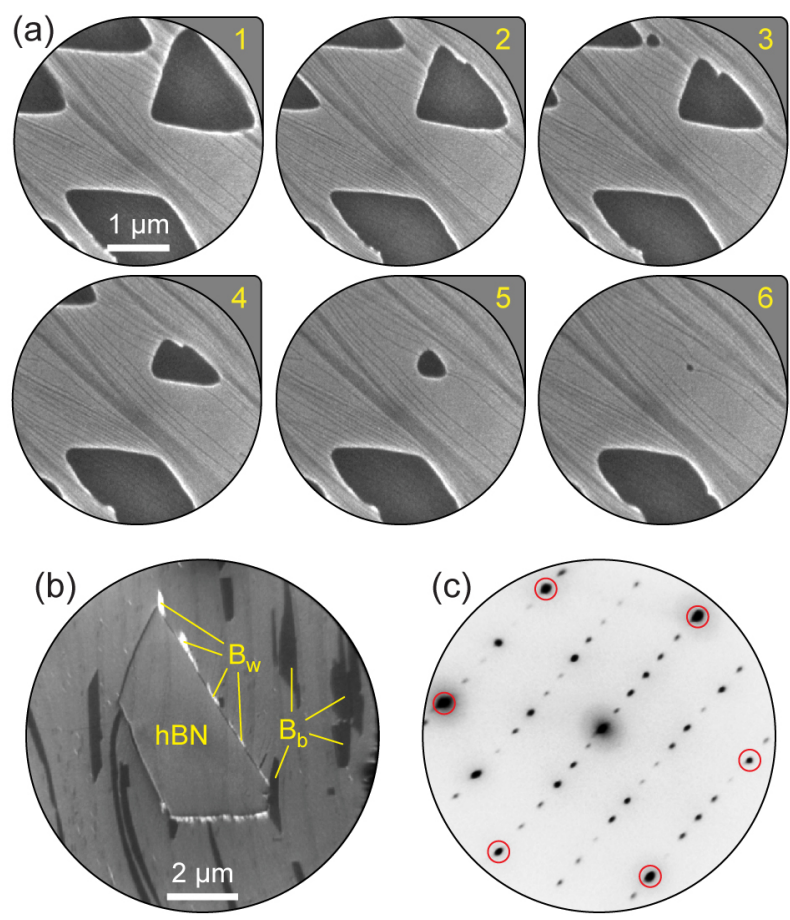

Figure 2. (a) Decomposition of $\mathrm{hBN}$ islands at $1100{ }^{\circ} \mathrm{C}$. LEEM images are taken in equidistant time intervals of $53 \mathrm{~s}$. $\mathrm{E}=16.6 \mathrm{eV}$. (b) hBN island surrounded by additional boron in the form of dark regions filling Ir terraces $\left(B_{b}\right)$ and white patches decorating $\mathrm{hBN}$ island edges $\left(\mathrm{B}_{\mathrm{w}}\right)$. Synthesis temperature was $1100{ }^{\circ} \mathrm{C}$, image recorded at room temperature. $\mathrm{E}=16.5 \mathrm{eV}$. (c) $\mu$-LEED pattern of $\mathrm{a} \mathrm{B}_{\mathrm{b}}$ region exhibiting a $(6 \times 2)$ superstructure with respect to Ir. Red circles indicate positions of first-order Ir spots. $\mathrm{E}=43 \mathrm{eV}$.

synthesis at $1100{ }^{\circ} \mathrm{C}$ and subsequent cooldown to room temperature. Beside a compact hBN island, extra material is visible in the form of dark, elongated features that predominantly follow the shape of Ir step edges, and irregular bright patches that decorate $\mathrm{hBN}$ island edges. We label this additional material as $\mathrm{B}_{\mathrm{b}}$ and $\mathrm{B}_{\mathrm{w}}$, respectively. $\mathrm{B}_{\mathrm{b}}$ regions appeared during cooldown at $\approx 950$ ${ }^{\circ} \mathrm{C}$, and they were also observed if cooldown was performed before any hBN nucleation or after hBN disintegration. We noticed that $B_{w}$ regions grew if the sample was kept at elevated synthesis temperature without dosing borazine and the $\mathrm{hBN}$ island they grew attached to remained unaltered. All this evidence suggests that the $\mathrm{B}_{\mathrm{b}}$ and $\mathrm{B}_{\mathrm{w}}$ regions are in fact reconstructed boron areas. $\mathrm{B}$ atoms are fed to those regions from the borazine gas phase and from disintegrated hBN islands, while the mechanism of $\mathrm{B}_{\mathrm{b}}$ and $\mathrm{B}_{\mathrm{w}}$ formation is either (i) diffusion of $B$ atoms across the Ir surface and eventual nucleation or (ii) dissolution of B atoms into Ir subsurface regions followed by segregation due to the decrease of solubility at lower temperatures. Therefore, the threshold temperature for significant disintegration of hBN on (111) face of Ir and the diffusion of B into the subsurface regions is 
$\approx 950{ }^{\circ} \mathrm{C}$, and a slightly lower temperature $\left(900{ }^{\circ} \mathrm{C}\right)$ was used for synthesis when hBN islands without excessive $\mathrm{B}$ regions surrounding them were desired. $\mu$-LEED analysis, enabling diffraction studies of sub-micrometer-sized areas on the sample surface, revealed that $B_{b}$ regions exhibit an adsorbate (not adlayer) form due to the relatively dilute $(6 \times 2)$ superstructure [see the diffraction pattern in Fig. 2(c)], and they were present on the surface in three equivalent $120^{\circ}$-rotated variants. $B_{w}$ regions exhibit a $(1 \times 1)$ (or possibly disordered) structure (not shown). We also note that $\mathrm{B}_{\mathrm{b}}$ and $\mathrm{B}_{\mathrm{w}}$ regions are easily etched away at elevated temperatures with oxygen.

In order to confirm our speculations on the chemical composition of $\mathrm{B}_{\mathrm{b}}$ and $\mathrm{B}_{\mathrm{w}}$ regions, AES was performed. A suitable area for AES was first found with SEM and is shown in Fig. 3(a). hBN islands appear dark and $\mathrm{B}_{\mathrm{b}}$ regions are visible as elongated, slightly lighter structures when compared with the surrounding bare Ir. The mode of contrast generation in SEM (which is different to the one in LEEM) does not enable imaging of the small $B_{w}$ regions attached to hBN. Because of this, $B_{w}$ regions were not accessible for Auger analysis. Three characteristic positions (bare $\mathrm{Ir}, \mathrm{hBN}$ and $\mathrm{B}_{\mathrm{b}}$ ) marked by colored dots (green, red and blue, respectively) were chosen and the resulting AES spectra are shown in Fig. 3(b), with a focus on the energy range where the main $B$ peak is expected to be found [45. A notable Ir peak is located in the same region and contributes to all recorded Auger spectra, which hinders our analysis. A larger peak of the hBN spectrum (red curve) as compared with the bare Ir spectrum (green curve) in the region indicated by arrow 1 is ascribed to the presence of $\mathrm{B}$ in $\mathrm{hBN}$. The excess of $B$ can also be read out from the hBN spectrum peak in the area indicated by arrow 2. The Auger spectrum of $\mathrm{B}_{\mathrm{b}}$ (blue curve) is more similar to bare Ir spectrum, and the two spectra are in principle indistinguishable in the region of arrow 1. However, a difference between them is found in the region of arrow 2 where the discernible peak of the $B_{b}$ region is visible. Although small, this difference between bare Ir and $\mathrm{B}_{\mathrm{b}}$ spectra was systematically found for different sets of spectra, and we therefore ascribe it to the presence of $\mathrm{B}$ in $\mathrm{B}_{\mathrm{b}}$ regions. One should keep in mind the concentration of $\mathrm{B}$ within the $\mathrm{B}_{\mathrm{b}}$ : it is approximately $1 / 12$ of the concentration within the $\mathrm{hBN}$ island (under the assumption of one $\mathrm{B}$ atom per $\mathrm{B}_{\mathrm{b}}$ unit cell), thus diminishing the intensity of the B Auger peaks by the same factor.

The disintegration of $\mathrm{hBN}$ and the presence of additional B species on the surface is complemented by several published studies of $\mathrm{hBN} / \operatorname{Ir}(111)$. From their XPS results, Usachov et al. found that $\mathrm{B}$ 1s and $\mathrm{N}$ 1s spectra intensities decrease rapidly if the sample is kept at temperatures higher than $1000{ }^{\circ} \mathrm{C}$ [29], ascribing this to hBN decomposition accompanied by $\mathrm{N}$ desorbtion and $\mathrm{B}$ diffusion to the Ir subsurface regions. Moreover, the authors find, besides the main peak assigned to $\mathrm{hBN}$,
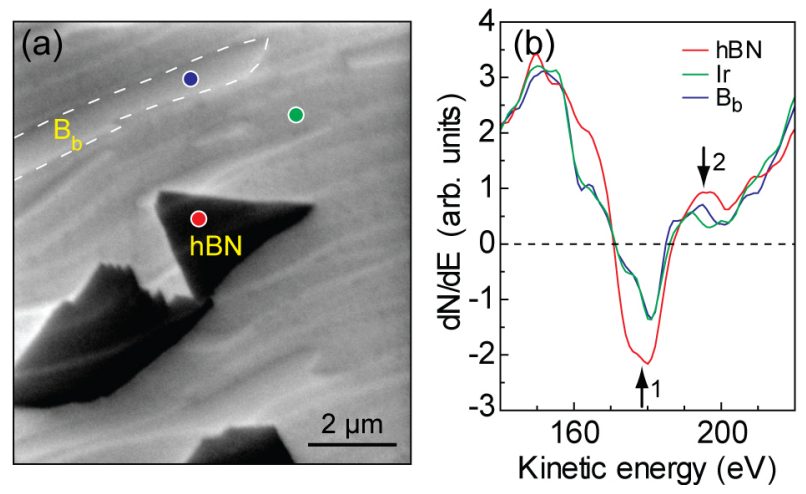

Figure 3. (a) SEM image of hBN islands (dark) on $\operatorname{Ir}(111)$ with elongated $B_{b}$ regions surrounding them (bright, one of the islands is indicated by a dashed line). Colored dots mark the positions where Auger spectra shown in (b) were recorded. (b) Auger spectra of an hBN island, bare Ir and $\mathrm{B}_{\mathrm{b}}$ region. Arrows indicate regions that enable identification of $\mathrm{B}$ on the sample (see text for details).

two additional B peaks that signify the existence of two additional forms of boron. In the same study, LEED images indicate a $(6 \times 2)$ superstructure in addition to the moiré diffraction spots, which is evidence for the presence of $\mathrm{B}_{\mathrm{b}}$ islands. Farwick zum Hagen et al. provided an atomistic view of $B_{w}$ islands via STM that exhibit a $(1 \times 1)$ structure 28 . There, the existence of $B_{w}$ islands was ascribed to the decomposition of $\mathrm{hBN}$ because the authors observed a similar structure in the inner parts of $\mathrm{hBN}$ islands. Therefore, the process of hBN disintegration as well as the formation of different B structures on the Ir surface has been evidenced by several experimental techniques and should be taken into account for optimal hBN synthesis, and considered as initial steps towards potential synthesis of borophene on iridium.

\section{B. Island shape and alignment with iridium}

We now turn to a more detailed analysis of individual hBN islands. After a CVD synthesis at $900{ }^{\circ} \mathrm{C}$, two basic island shapes were found on Ir surface: triangular and trapezoidal, as shown in Fig. 4(a). This is particularly the case in the initial stages of growth when the islands are small and their shape (i.e., edge straightness and angles between the edges) is not much affected by the Ir step morphology. Typical island size ranges from 1 to $2 \mu \mathrm{m}$ and sides of neighboring triangles and trapezoids are parallel or nearly parallel, indicating alignment with respect to the substrate and the existence of a preferred edge type. The island densities of both triangular and trapezoidal islands were roughly equal. Moreover, the two island types are rotated by $180^{\circ}$ with respect to each other, as is inferred from their diffraction patterns shown in Fig. 4(b). 
High-symmetry crystal directions, which are easily determined in LEED, can be mapped to real-space directions in LEEM images, and this allows for the determination of the $\mathrm{hBN}$ island edge type. It is found that all edges (for both triangles and trapezoids) are of the zig-zag (ZZ) type under the assumption that no edge reconstruction takes place (as has been proposed theoretically for hBN on metals [46]). Indeed, the $\mathrm{ZZ}$ edge type has also been confirmed in STM experiments, and borontermination has been suggested based on the DFT calculations [28, 47]. Deviations from ZZ edges occur when islands grow bigger and initial straight edges, mutually connected at $60^{\circ}$ or $120^{\circ}$, are lost. The mere observation of triangular island shapes also suggests a ZZ edge type because $\mathrm{ZZ}$ edge configuration in hexagonal 2D materials with bi-elemental basis enables the presence of one atomic species on the edge, the one which provides an edge of lower energy. In the case of $\mathrm{G}$ growth, initial small islands are often hexagonal because of mono-elemental basis.

The existence of two $180^{\circ}$-rotated variants of $\mathrm{hBN}$ islands on $\operatorname{Ir}(111)$ was reported before, including the synthesis recipe enabling prevalence of one orientation over the other [27, 28]. Also, triangular islands of two orientations have been reported for hBN on strongly interacting substrates such as $\mathrm{Ni}(111)$ [4], $\mathrm{Rh}(111)$ [15], $\mathrm{Ru}(0001)$ 49 and $\mathrm{Co}(0001)$ 34. Theoretical calculations for systems without the moiré structure showed that the two orientations stem from the two possible stable configurations of hBN on metals 25, 50. In both of them, $\mathrm{N}$ atoms are located on top of the Ir atoms $\left(\mathrm{N}_{\text {top }}\right)$, while $\mathrm{B}$ atoms can be either in the hcp $\left(\mathrm{B}_{\mathrm{hcp}}\right)$ of fcc $\left(\mathrm{B}_{\mathrm{fcc}}\right)$ sites, leading to the formation of two $180^{\circ}$-rotated domains. However, for systems that develop the moiré structure, such as $\mathrm{hBN} / \mathrm{Ir}(111)$, the registry between $\mathrm{N}$ and $\mathrm{B}$ atoms with respect to Ir atoms changes across the moiré unit cell. But even in such cases, two $180^{\circ}$-rotated orientations of the hBN layer are preferred, especially if island edge interaction with the substrate is considered to be dominant in total binding [28, 51].

One of our AFM measurements is shown in Fig. 4(c) where individual triangular and trapezoidal $\mathrm{hBN}$ islands as well as Ir steps are visible. The short base of the trapezoidal island always forms in the step-up direction of Ir. It should be noted that because the trapezoidal islands have two ZZ edges that are parallel, they must contain different types of edge atoms. Therefore, trapezoidal islands form a ZZ edge of the opposite composition in the step-up direction as compared with all other edges. Because this behavior is absent for triangular islands, we speculate that it is a result of an energetic balance between the two ZZ edge types (N- and B-terminated) and different interaction of the two island types with the substrate, in particular with the substrate step edges. Indeed, STM measurements 28 have shown that both island types are triangular in the initial phases of growth when their dimensions do not exceed typical Ir terrace widths, indicating the importance of Ir steps for the overall island shape. The issue of the binding of hBN islands to Ir will be addressed in more detail in the next section.

The rotational registry of $\mathrm{hBN}$ islands synthesized at $900{ }^{\circ} \mathrm{C}$ was investigated by $\mu$-LEED analysis. With the aid of the moiré structure, relative rotations of hexagonal $2 \mathrm{D}$ overlayers with respect to Ir are effectively magnified [52] and can therefore be determined with high precision. For hBN on $\operatorname{Ir}(111)$, this magnification is $\sim 11.1$ times, as can be calculated from the lattice constant values given in Subsection 3.1. By scanning over many islands, small rotations in the sub-degree region were found. An example is given in Fig. 4(d) for three neighboring islands exhibiting small rotations $\left(0^{\circ}\right.$ is defined as an alignment of hBN ZZ direction and dense packed atomic rows of Ir). A small angle misalignment between $\mathrm{hBN}$ and Ir lattices was reported before for $\mathrm{hBN} / \operatorname{Ir}(111)$ [28, 42, and is also known to be present in $\mathrm{G}$ on $\operatorname{Ir}(111)$ [52, 53. The major factor influencing orientational quality of both 2D materials is the synthesis temperature [29, 54], and in our case it could be improved at the expense of the $\mathrm{B}_{\mathrm{b}}$ and $\mathrm{B}_{\mathrm{w}}$ regions formation.

\section{Work function and interaction with iridium}

Basic information about the electronic structure of the triangular and trapezoidal islands was obtained from the combination of IV-LEEM spectroscopy and PEEM. In Fig. 5(a), three normalized bright field IV-LEEM spectra taken from bare $\mathrm{Ir}$ and the two $\mathrm{hBN}$ island types are shown. By looking at the drop in electron reflectivity when increasing the electron energy, it is obvious that the examined areas have different work functions (WFs) [55]. We estimate the difference in the WF between Ir and triangular hBN island to be $0.98 \mathrm{eV}$, while it is larger for the trapezoidal island and amounts to $1.08 \mathrm{eV}$. In other words, trapezoidal islands exhibit a WF that is 0.1 $\mathrm{eV}$ lower that the WF of triangular islands. These values were obtained by performing a linear fit to the reflectivity shoulders and then determining their relative offsets at a reflectivity value of 0.8 . By taking the WF of bare Ir to be $5.76 \mathrm{eV}$ [56], hBN triangles and trapezoids have WFs of 4.78 and $4.68 \mathrm{eV}$, respectively.

These values are somewhat lower that the ones reported in an $I(z)$ spectroscopy study of $\mathrm{hBN} / \operatorname{Ir}(111)$ by Schultz et al. 42. In the same work, the authors showed that more strongly interacting areas of the $\mathrm{hBN} / \operatorname{Ir}(111)$ moiré cell exhibit larger WF. Similarly, from IV-LEEM measurements of $\mathrm{G}$ on $\operatorname{Ir}(111)$, Starodub et al. [57] found that more strongly bound rotational variant of $G$ has a higher WF. Therefore, we speculate that of the two hBN island types, triangular ones are more strongly bound to Ir. A difference in binding strength, although amounting only to $2.8 \mathrm{meV}$ per hBN unit cell, has also been 

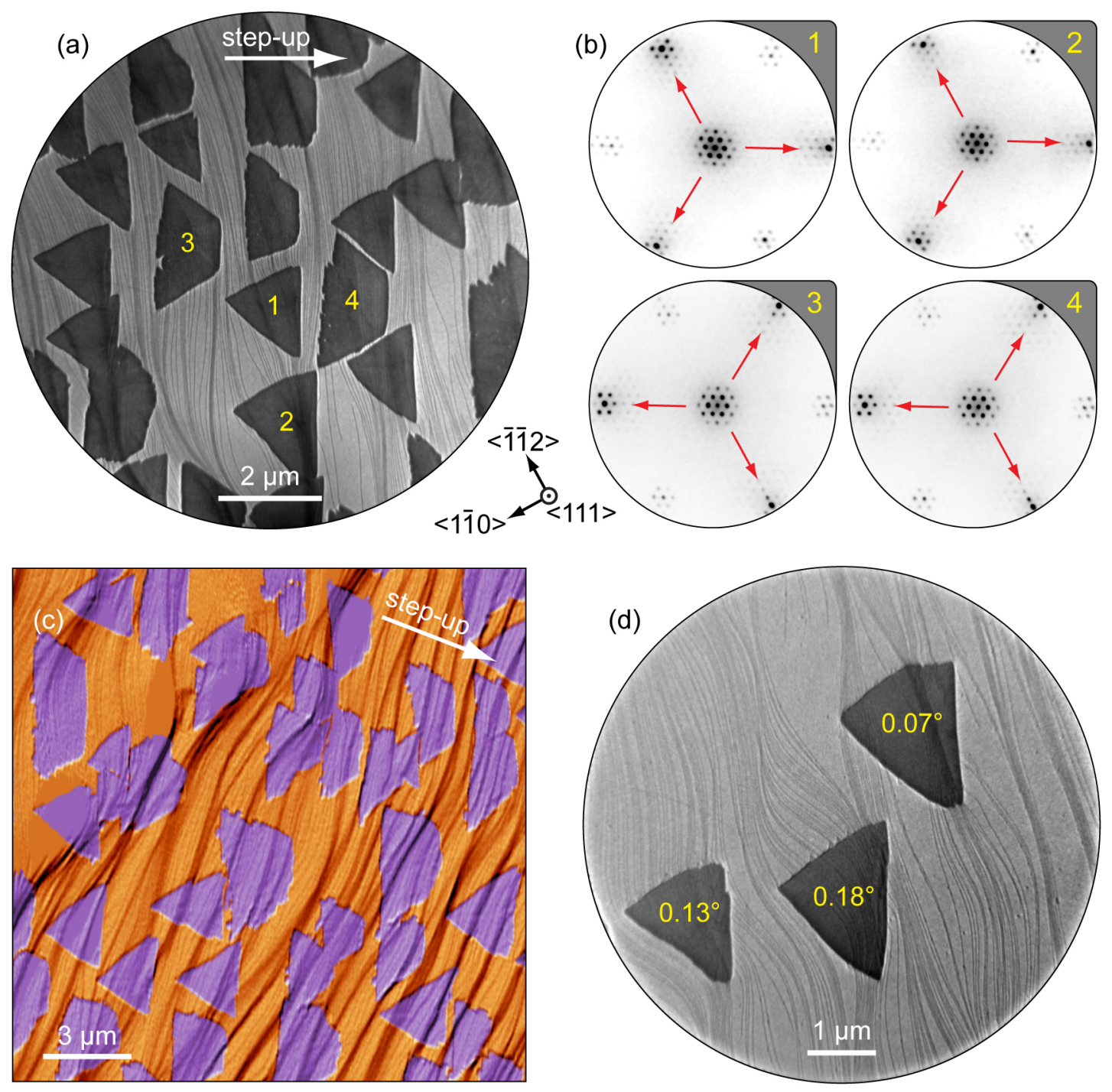

Figure 4. (a) LEEM image of hBN islands on $\operatorname{Ir}(111)$. Two island shapes, triangular and trapezoidal, are distinguished. Thin wavy lines extending vertically are Ir steps. An arrow points in the step-up direction of Ir surface. E $=16.2 \mathrm{eV}$. (b) $\mu$-LEED patterns of four $\mathrm{hBN}$ islands marked in panel (a), enabling identification of their relative $180^{\circ}$ rotation. Arrows point to the groups of diffraction spots exhibiting higher intensity. $\mathrm{E}=40 \mathrm{eV}$. (c) AFM image of hBN islands on $\operatorname{Ir}(111)$. A first derivative of topography is shown and $\mathrm{hBN}$ islands (along with some $\mathrm{B}_{\mathrm{w}}$ regions) have been colored in purple for clarity. Wavy lines are Ir steps; an arrow points in the step-up direction. (d) LEEM image of three triangular hBN islands exhibiting the noted rotations with respect to Ir, as inferred from the analysis of their $\mu$-LEED patterns (not shown). Ir steps are visible as thin wavy lines. $\mathrm{E}=17.1 \mathrm{eV}$. Crystallographic directions of Ir noted in the center of the figure apply to all panels. All images were recorded at room temperature.

suggested based on the DFT calculations [28].

The binding inequivalence speculation is further supported by a PEEM measurement shown in Fig. 5(b). It demonstrates that triangular and trapezoidal islands exhibit different photoemission intensities, which in fact reflect differences in the electronic structure of the $\mathrm{hBN} / \operatorname{Ir}(111)$ system as a whole. The first factor that can contribute to this difference is the $\mathrm{WF}$ variation. The second factor to be considered is the coherence of the Ir surface state at the $\Gamma$ point [58], which is the most probable source of photoelectrons in PEEM images, because it remains present on the surface after $\mathrm{hBN}$ formation [29]. A strong hBN-Ir interaction can potentially cause quenching of the surface state [42, which would reduce the photoemission yield in PEEM. Therefore, both IVLEEM and PEEM measurements suggest stronger interaction of the triangular hBN islands with Ir, as opposed to the trapezoidal ones. Overall, different binding of the two island types could be the source of different growth modes and consequently, in combination with substrate morphology, different island shapes. Trapezoidal islands have been observed for $\mathrm{hBN}$ on $\mathrm{Pt}(111)$ where binding 


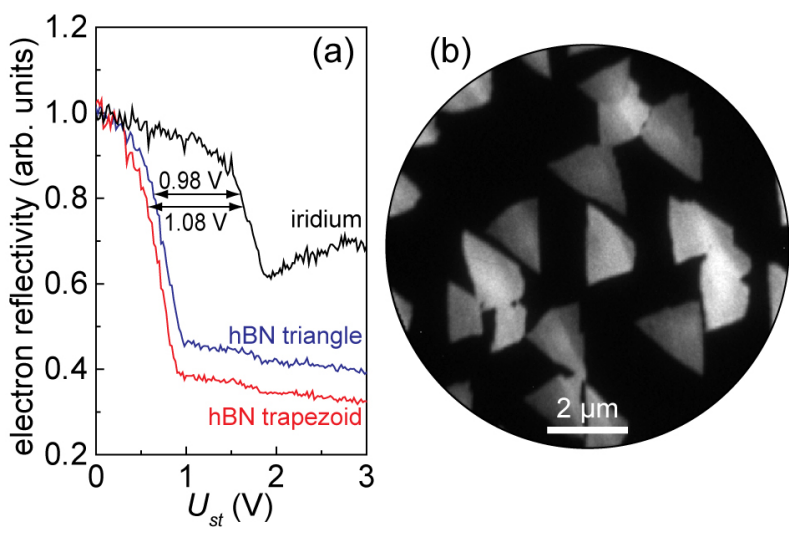

Figure 5. (a) Bright field IV-LEEM spectra taken form Ir (black curve) and the two types of hBN islands (blue and red curves). Both island types display a reduction of the WF as visible from the offsets in start voltage $\left(U_{s t}\right)$, which are indicated by double-headed arrows. (b) PEEM image of hBN islands on Ir. Due to the WF modifications, the Ir surface remains dark while the two island types exhibit different photoemission intensity (see text for details).

to the substrate is weak, but their origin was not discussed [31]. In contrast, strongly interacting substrates such as Ru or Rh do not show signs of trapezoidal islands growth. Therefore, there is an intimate relation between the strength of hBN-substrate interaction (especially at the step edges) and the final shape of the islands.

Finally, we note that photoemission intensity variations are visible within individual islands in Fig. 5(b). Comparison with the corresponding LEEM images of the same area (not shown) reveals that this change is related to the step morphology of Ir, more precisely step bunches. Knowing that the emission angle of the photoelectrons originating from different areas on the islands does not change sufficiently to cause such changes, intensity variations are a sign of binding strength modification within a single island due to the stronger interaction with Ir step edges, an effect that is known to be present in G on Ir [59].

\section{CONCLUSION}

Atomically thin hBN islands of excellent crystal quality and size up to $\sim 10 \mu \mathrm{m}$ can be easily synthesized on the (111) surface of Ir via CVD process with borazine as a precursor. Two types of hBN islands have been observed: triangular and trapezoidal, which are rotated by $180^{\circ}$ with respect to each other and which have $\mathrm{ZZ}$ edges. Both island types have the same atom species on all edges (presumably boron, based on the DFT calculations from Ref. [28]) except for the short base of the trapezoidal islands where an edge of opposite composition must exist. These structural results are illustrated in Fig. 6. Based

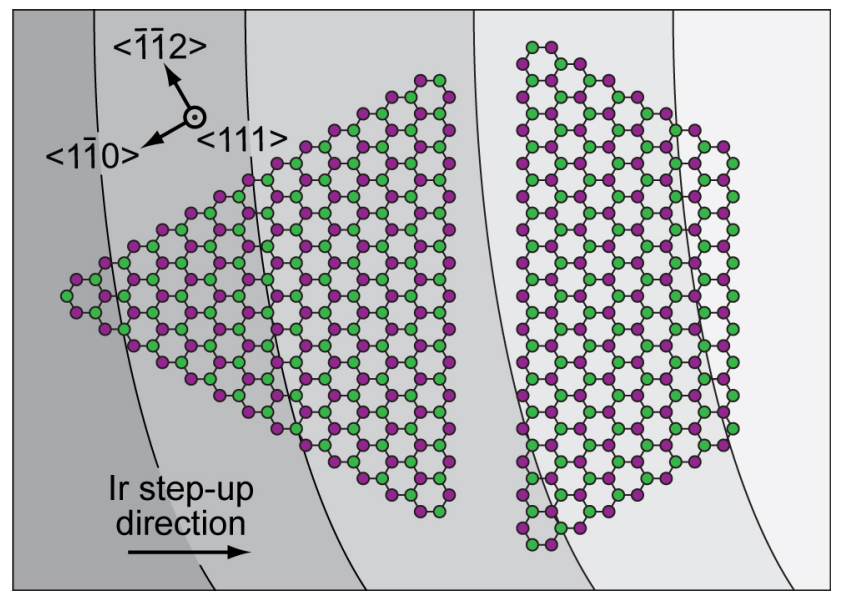

Figure 6. Schematic models of the triangular and trapezoidal hBN islands found on $\operatorname{Ir}(111)$, which are rotated by $180^{\circ}$ with respect to each other. All island edges are of the $\mathrm{ZZ}$ type and of the same composition, except for the short base of the trapezoidal island. Black lines extending vertically are the Ir step edges. Crystallographic directions and the stepup direction of Ir are marked in the top-left and bottom-left corner, respectively.

on photoemission and spectroscopic data, we conclude that different shapes of the islands originate from different interactions with Ir. Moreover, Ir steps are crucial for the growth of hBN because they break the mirror symmetry of the $180^{\circ}$-rotated islands, which triggers different growth modes of hBN. This results in either triangular or trapezoidal islands, and the short base of the trapezoidal islands always forms in the step-up direction of the Ir surface. For synthesis temperatures lower than $\approx 950{ }^{\circ} \mathrm{C}, \mathrm{hBN}$ disintegration and dissolution of $\mathrm{B}$ in the Ir subsurface areas are greatly inhibited and hence such temperatures should be used in order to avoid formation of boron reconstructed regions. In these regions, boron atoms either fill large areas of $\operatorname{Ir}$ terraces $\left(\mathrm{B}_{\mathrm{b}}\right.$ regions) or form small irregular patches attached to $\mathrm{hBN}$ islands ( $\mathrm{B}_{\mathrm{w}}$ regions). This provides new possibilities for the production of other novel boron-based 2D materials, such as borophene. Overall, our results give new insights into CVD synthesis of hBN and can be used to steer future production of this important $2 \mathrm{D}$ material.

\section{ACKNOWLEDGMENTS}

Marko Kralj is acknowledged for critical reading of the manuscript and useful discussions. M.P. would like to thank the Alexander von Humboldt Foundation for financial support. 


\section{REFERENCES}

marin.petrovic@gast.uni-due.de Permanent address: Center of Excellence for Advanced Materials and Sensing Devices, Institute of Physics, Bijenika 46, 10000 Zagreb, Croatia

[1] M. Xu, T. Liang, M. Shi, and H. Chen, Chem. Rev. 113, 3766 (2013).

[2] A. Pakdel, Y. Bando, and D. Golberg, Chem. Soc. Rev. 43, 934 (2014).

[3] G. Cassabois, P. Valvin, and B. Gil, Nat. Photonics 10, $262(2016)$.

[4] C. R. Dean, A. F. Young, I. Meric, C. Lee, L. Wang, S. Sorgenfrei, K. Watanabe, T. Taniguchi, P. Kim, K. L. Shepard, and J. Hone, Nat. Nanotechnol. 5, 722 (2010).

[5] M. S. Bresnehan, M. J. Hollander, M. Wetherington, M. LaBella, K. A. Trumbull, R. Cavalero, D. W. Snyder, and J. A. Robinson, ACS Nano 6, 5234 (2012).

[6] M. P. Levendorf, C.-J. Kim, L. Brown, P. Y. Huang, R. W. Havener, D. A. Muller, and J. Park, Nature 488, 627 (2012).

[7] G.-H. Lee, X. Cui, Y. D. Kim, G. Arefe, X. Zhang, C.-h. Lee, F. Ye, K. Watanabe, T. Taniguchi, P. Kim, and J. Hone, ACS Nano 9, 7019 (2015).

[8] K. Watanabe, T. Taniguchi, and H. Kanda, Nat. Mater. 3, 404 (2004).

[9] M. Corso, W. Auwärter, M. Muntwiler, A. Tamai, T. Greber, and J. Osterwalder, Science 303, 217 (2004).

[10] F. Schulz, R. Drost, S. K. Hämäläinen, and P. Liljeroth, ACS Nano 7, 11121 (2013).

[11] W. Auwärter, T. Kreutz, T. Greber, and J. Osterwalder, Surf. Sci. 429, 229 (1999).

[12] M. Morscher, M. Corso, T. Greber, and J. Osterwalder, Surf. Sci. 600, 3280 (2006).

[13] A. Goriachko, Y. He, M. Knapp, H. Over, M. Corso, T. Brugger, S. Berner, J. Osterwalder, and T. Greber, Langmuir 23, 2928 (2007).

[14] E. Cavar, R. Westerström, A. Mikkelsen, E. Lundgren, A. Vinogradov, M. L. Ng, A. Preobrajenski, A. Zakharov, and N. Mårtensson, Surf. Sci. 602, 1722 (2008).

[15] G. Dong, E. B. Fourré, F. C. Tabak, and J. W. M. Frenken, Phys. Rev. Lett. 104, 096102 (2010).

[16] F. Müller, S. Hüfner, H. Sachdev, R. Laskowski, P. Blaha, and K. Schwarz, Phys. Rev. B 82, 113406 (2010).

[17] F. Orlando, R. Larciprete, P. Lacovig, I. Boscarato, A. Baraldi, and S. Lizzit, The Journal of Physical Chemistry C 116, 157 (2012).

[18] S. Joshi, D. Ecija, R. Koitz, M. Iannuzzi, A. P. Seitsonen, J. Hutter, H. Sachdev, S. Vijayaraghavan, F. Bischoff, K. Seufert, J. V. Barth, and W. Auwärter, Nano Lett. 12, 5821 (2012).

[19] J. Wintterlin and M.-L. Bocquet, Surface Science 603, 1841 (2009).

[20] E. Celasco, G. Carraro, M. Smerieri, L. Savio, M. Rocca, and L. Vattuone, The Journal of Chemical Physics 146, 104704 (2017).

[21] A. B. Preobrajenski, A. S. Vinogradov, and N. Mårtensson, Surf. Sci. 582, 21 (2005).
[22] A. B. Preobrajenski, A. S. Vinogradov, M. L. Ng, E. Ćavar, R. Westerström, A. Mikkelsen, E. Lundgren, and N. Mårtensson, Phys. Rev. B 75, 245412 (2007).

[23] R. Laskowski and P. Blaha, Phys. Rev. B 81, 075418 (2010).

[24] A. B. Preobrajenski, M. A. Nesterov, M. L. Ng, A. S. Vinogradov, and N. Mårtensson, Chem. Phys. Lett. 446, 119 (2007).

[25] R. Laskowski, P. Blaha, and K. Schwarz, Phys. Rev. B 78, 045409 (2008).

[26] M. Kralj, I. Pletikosić, M. Petrović, P. Pervan, M. Milun, A. T. N'Diaye, C. Busse, T. Michely, J. Fujii, and I. Vobornik, Phys. Rev. B 84, 075427 (2011).

[27] F. Orlando, P. Lacovig, L. Omiciuolo, N. G. Apostol, R. Larciprete, A. Baraldi, and S. Lizzit, ACS Nano 8, 12063 (2014).

[28] F. H. Farwick zum Hagen, D. M. Zimmermann, C. C. Silva, C. Schlueter, N. Atodiresei, W. Jolie, A. J. Martínez-Galera, D. Dombrowski, U. A. Schröder, M. Will, P. Lazić, V. Caciuc, S. Blügel, T.-L. Lee, T. Michely, and C. Busse, ACS Nano 10, 11012 (2016).

[29] D. Usachov, A. Fedorov, O. Vilkov, V. K. Adamchuk, L. V. Yashina, L. Bondarenko, A. A. Saranin, A. Grüneis, and D. V. Vyalikh, Phys. Rev. B 86, 155151 (2012).

[30] A. J. Mannix, X.-F. Zhou, B. Kiraly, J. D. Wood, D. Alducin, B. D. Myers, X. Liu, B. L. Fisher, U. Santiago, J. R. Guest, M. J. Yacaman, A. Ponce, A. R. Oganov, M. C. Hersam, and N. P. Guisinger, Science 350, 1513 (2015).

[31] Y. Zhang, X. Weng, H. Li, H. Li, M. Wei, J. Xiao, Z. Liu, M. Chen, Q. Fu, and X. Bao, Nano Lett. 15, 3616 (2015).

[32] A. Goriachko, A. A. Zakharov, and H. Over, The Journal of Physical Chemistry C 112, 10423 (2008).

[33] P. Sutter, J. Lahiri, P. Albrecht, and E. Sutter, ACS Nano 5, 7303 (2011).

[34] C. M. Orofeo, S. Suzuki, H. Kageshima, and H. Hibino, Nano Res. 6, 335 (2013).

[35] K. K. Kim, A. Hsu, X. Jia, S. M. Kim, Y. Shi, M. Hofmann, D. Nezich, J. F. Rodriguez-Nieva, M. Dresselhaus, T. Palacios, and J. Kong, Nano Lett. 12, 161 (2012).

[36] A. T. N'Diaye, R. van Gastel, A. J. Martínez-Galera, J. Coraux, H. Hattab, D. Wall, F.-J. Meyer zu Heringdorf, M. Horn-von Hoegen, J. M. Gómez-Rodríguez, B. Poelsema, C. Busse, and T. Michely, New J. Phys. 11, 113056 (2009).

[37] M. Petrović, J. T. Sadowski, A. Šiber, and M. Kralj, Carbon 94, 856 (2015).

[38] C. Lee, X. Wei, J. W. Kysar, and J. Hone, Science 321, 385 (2008).

[39] L. Song, L. Ci, H. Lu, P. B. Sorokin, C. Jin, J. Ni, A. G. Kvashnin, D. G. Kvashnin, J. Lou, B. I. Yakobson, and P. M. Ajayan, Nano Lett. 10, 3209 (2010).

[40] J. W. Arblaster, Platin. Met. Rev. 54, 93 (2010).

[41] W. Paszkowicz, J. Pelka, M. Knapp, T. Szyszko, and S. Podsiadlo, Appl. Phys. A: Mater. Sci. Process. 75, 431 (2002).

[42] F. Schulz, R. Drost, S. K. Hämäläinen, T. Demonchaux, A. P. Seitsonen, and P. Liljeroth, Phys. Rev. B 89, 235429 (2014).

[43] J. C. Cornish and N. R. Avery, Surf. Sci. 235, 209 (1990).

[44] I. Zeiringer, X. Cheng, X.-Q. Chen, E. Bauer, G. Giester, and P. F. Rogl, Science China Materials 58, 649 (2015).

[45] P. Sutter and E. Sutter, APL Materials 2, 092502 (2014). 
[46] Z. Zhang, Y. Liu, Y. Yang, and B. I. Yakobson, Nano Lett. 16, 1398 (2016).

[47] M. Liu, Y. Li, P. Chen, J. Sun, D. Ma, Q. Li, T. Gao, Y. Gao, Z. Cheng, X. Qiu, Y. Fang, Y. Zhang, and Z. Liu, Nano Lett. 14, 6342 (2014).

[48] W. Auwärter, M. Muntwiler, J. Osterwalder, and T. Greber, Surf. Sci. 545, L735 (2003).

[49] J. Lu, P. S. E. Yeo, Y. Zheng, H. Xu, C. K. Gan, M. B. Sullivan, A. Castro Neto, and K. P. Loh, J. Am. Chem. Soc. 135, 2368 (2013).

[50] G. B. Grad, P. Blaha, K. Schwarz, W. Auwärter, and T. Greber, Phys. Rev. B 68, 085404 (2003).

[51] L. Camilli, E. Sutter, and P. Sutter, 2D Materials 1, 025003 (2014).

[52] A. T. N'Diaye, J. Coraux, T. N. Plasa, C. Busse, and T. Michely, New J. Phys. 10, 043033 (2008).
[53] J. Coraux, A. T. N'Diaye, C. Busse, and T. Michely, Nano Lett. 8, 565 (2008).

[54] H. Hattab, A. T. N'Diaye, D. Wall, G. Jnawali, J. Coraux, C. Busse, R. van Gastel, B. Poelsema, T. Michely, F.-J. Meyer zu Heringdorf, and M. Hornvon Hoegen, Appl. Phys. Lett. 98, 141903 (2011).

[55] M. Babout, C. Guittard, M. Guivarch, R. Pantel, and M. Bujor, J. Phys. D: Appl. Phys. 13, 1161 (1980).

[56] R. Strayer, W. Mackie, and L. Swanson, Surf. Sci. 34, 225 (1973).

[57] E. Starodub, A. Bostwick, L. Moreschini, S. Nie, F. E. Gabaly, K. F. McCarty, and E. Rotenberg, Phys. Rev. B 83, 125428 (2011).

[58] D. Niesner, T. Fauster, J. I. Dadap, N. Zaki, K. R. Knox, P.-C. Yeh, R. Bhandari, R. M. Osgood, M. Petrović, and M. Kralj, Phys. Rev. B 85, 081402 (2012).

[59] I. Šrut Rakić, M. Kralj, W. Jolie, P. Lazić, W. Sun, J. Avila, M.-C. Asensio, F. Craes, V. Mikšić Trontl, C. Busse, and P. Pervan, Carbon 110, 267 (2016). 